\section{Arbolado urbano. Estudio de caso: Reparto Hermanos Cruz, Pinar del Río, Cuba.}

\author{
Marta Margarita Bonilla Vichot ${ }^{1}$, Elizabeth Crespo \\ Paredes y Darien Felipe Medina Bonilla \\ ${ }^{1}$ Universidad de Pinar del Río, Cuba \\ mbon@upr.edu.cu
}

\section{Urban Woodland. Case study: County Hermanos} Cruz, Pinar del Río, Cuba.

Recibido: $17 / 06 / 2019$

Aceptado: $20 / 11 / 2019$

Publicado: $27 / 12 / 2019$

\title{
RESUMEN
}

La calidad de vida de los pobladores de una zona urbana depende, en gran medida, del estado y la abundancia de espacios verdes existentes en ella o en sus proximidades. La investigación que se presenta recoge el estudio realizado en las áreas verdes del reparto Hermanos Cruz, municipio Pinar del Río, con el objetivo de caracterizar el arbolado urbano a partir de la composición, diversidad y percepción de los pobladores sobre sus funciones. Se procedió a seleccionar 11 áreas. A partir de la Norma Cubana de Áreas verdes (NC 677-1:2009), se clasificaron las mismas por su ubicación y función; se inventariaron las correspondientes a parques e instalaciones públicas, así como el arbolado viario. En este sentido, se identificaron: árboles de riesgo; principales conflictos; índice de riquezas; frecuencia relativa; y abundancia relativa; y se aplicaron encuestas a los pobladores para conocer su percepción sobre el arbolado urbano. Como resultados del estudio se obtuvo que, en la distribución de las especies por hábitos de crecimiento, se observa un predominio de los árboles, destacándose las familias Bignoniaceae, Malvaceae, Meliaceae, Caesalpinaceae, Annonaceae y Arecaceae como las más representadas. Con respecto al origen predominan las especies introducidas tanto para los árboles como para los arbustos. La riqueza de especies presenta una variación entre las diferentes áreas. Las personas consideran necesaria la presencia de los árboles en las ciudades y demuestran conocimientos acerca del arbolado en su entorno citadino, en cuanto a sus funciones y beneficios. Se realizan propuestas para incrementar las especies autóctonas.

PALABRAS CLAVE: árboles de riesgo, áreas verdes, diversidad, riqueza de especies.

\section{ABSTRACT}

The quality of life of the inhabitants of an urban area depends, to a large extent, on the state and the abundance of green spaces existing in it or in its vicinity. The research presented includes the study carried out in the green areas of the cast Hermanos Cruz, municipality Pinar del Río, with the aim of characterizing the urban trees based on the composition, diversity and perception of the inhabitants about their functions. 11 areas were selected. From the Cuban Standard of Green Areas (NC 677-1: 2009), they were classified according to their location and function; those corresponding to parks and public facilities were inventoried, as well as the tree-lined road. In this regard, the following were identified: risk trees; main conflicts; wealth index; relative frequency; and relative abundance; and surveys were applied to the settlers to know their perception of urban trees. As results of the study it was obtained that, in the distribution of the species by growth habits, a predominance of the trees is observed, highlighting the families Bignoniaceae, Malvaceae, Meliaceae, Caesalpinaceae, Annonaceae and Arecaceae as the most represented. Regarding the origin, the species introduced for both trees and shrubs predominate. Species richness presents a variation between the different 
areas. People consider the presence of trees in cities necessary and demonstrate knowledge about woodland in their city environment, in terms of their functions and benefits. Proposals are made to increase native species.

KEYWORDS: diversity, green areas, risk trees, species richness.

\section{INTRODUCCIÓN}

El uso de la vegetación dentro de las ciudades se ha distinguido por responder a necesidades relacionadas con las características climáticas, socioculturales y económicas de las diferentes zonas del planeta. Estas prácticas, muy asociadas a la calidad ambiental y de vida, han cobrado gran importancia en los últimos años debido, sobre todo, al reconocimiento de los múltiples valores de las plantas, que preservados y mejorados constituyen una nueva manera de enfocar el urbanismo (Castillo y Ferro, 2015).

Coyula (1985), citado por Castillo y Ferro (2015) expresa que dentro de las formas en que aparecen las áreas verdes en la ciudad, el arbolado viario constituye la más significativa, debido a la directa relación que establece el árbol con el espacio de las calles y la población en general. Se suman a los verdes urbanos las áreas que se encuentran en instituciones, edificios públicos, parques, etc.

Diferentes autores como Castillo y Ferro (2015), Chang (2015), Pérez (2014), Sosa-López et. al (2011) y Álvarez de Zayas (2008), destacan las características de las especies más frecuentes, abundancia, composición del arbolado en diferentes ciudades del país, e incluyen además, el origen y valoraciones económicas a partir de sus funciones, entre otros aspectos, que permiten trazar pautas para estructurar de forma armónica la funcionabilidad y la belleza del arbolado en las ciudades.

El presente estudio tuvo como objetivo caracterizar el arbolado urbano a partir de la composición, diversidad y percepción de los pobladores sobre sus funciones; con la intención de evaluar el comportamiento de dichas variables en el reparto Hermanos Cruz, para establecer acciones futuras que permitieran incrementar la educación ambiental y el conocimiento de la importancia del arbolado urbano, en virtud de favorecer su protección y la incorporación de especies autóctonas en los diferentes espacios verdes, bajo el precepto de la silvicultura urbana: El árbol adecuado en el lugar adecuado.

\section{MATERIALES Y MÉTODOS}

El área de estudio pertenece al municipio Pinar del Río, consejo popular Hermanos Cruz, y se encuentra situada al Noreste de la ciudad de Pinar del Río; limita al Noroeste con el consejo popular 10 de octubre, al Sur con el reparto Carlos Manuel y al Este con Las Ovas.

Como aspectos climáticos se destacan la presencia de un periodo poco lluvioso con dos momentos bien definidos (noviembre y de enero - abril) y un periodo muy lluvioso (mayo - octubre). El total anual de precipitaciones es de $1554.5 \mathrm{~mm}$ y la temperatura media anual es de $24.2^{\circ} \mathrm{C}$; la máxima absoluta es de $36.3^{\circ} \mathrm{C}$ y la mínima absoluta es de $5.1^{\circ} \mathrm{C}$.

Para el desarrollo del trabajo se efectuó un recorrido por el reparto Hermanos Cruz, y se ubicaron un total de 11 áreas, consideradas cada una como parcelas de muestreo.

Atendiendo a la NC 53-05:78 Áreas verdes (Ministerio de Ciencia, Tecnología y Medio Ambiente [CITMA], 1978), se clasificaron estas para el muestreo en los verdes de uso común, incorporados a los espacios públicos de uso general por parte de la población; y los verdes de uso local limitado, 
vinculados a determinados conjuntos de edificios y espacios, la protección de suelos, el control climático, la protección ambiental, la producción de alimentos y la defensa del país.

Se procedió a hacer un inventario del arbolado viario y áreas verdes correspondientes a parques e instalaciones públicas, donde se identificaron las plantas presentes en ellas teniendo en cuenta:

- Cantidad de individuos; nombre científico; nombre vulgar y familias botánicas representativas.

- Clasificación de los árboles de acuerdo a su origen geográfico (endémicas, autóctonas, o introducidas).

Para su identificación se utilizaron los criterios de Sablón (1987), Bisse (1988) y Acevedo- Rodríguez y Strong (2012).

Atendiendo a la norma ramal NC 93-06-101:87 (CITMA, 1987), se clasificaron por sus hábitos de crecimiento a las especies en; Árboles, arbustos, estipitadas y herbáceas. Además, se clasificaron los diferentes tipos de plantaciones: bosquecillo, rodal, setos, setos podados y canteros, conformadas por la presencia de árboles o arbustos en las áreas evaluadas. Se determinaron los parámetros ecológicos a partir de los elementos estructurales: Abundancia relativa y frecuencia relativa en las distintas áreas.

Se determinó la frecuencia relativa y abundancia relativa de las principales especies presentes en las diferentes zonas, según las siguientes ecuaciones:

$$
\begin{gathered}
\mathrm{Ar}=\frac{\text { Número de árboles por especie }}{\text { Número de árboles totales }} \times 100 \\
\mathrm{Fr}=\frac{\text { Frecuencia absoluta de una especie }}{\text { Total de frecuencia absoluta }} \times 100
\end{gathered}
$$

Se estableció el índice de riqueza para los árboles presentes en las áreas verdes, el cual representa el número total de especies en una comunidad.

Se utilizó la metodología de Iguiñiz (2007) para evaluar los posibles riesgos que presentaban los árboles a partir del estado fitosanitario de las ramas, fuste y raíces, con la utilización del método de evaluación visual del árbol (EVA) y se clasificó además, el estado general los mismos: enfermo, moribundo y muerto.

De igual forma, se evaluó la estructura y estado del árbol a partir de daños biomecánicos, con énfasis en los siguientes aspectos: presencia de plagas; daños abióticos; pudrición en raíces, fustes y ramas; presencia de heridas; brotes de raíz; cavidades en troncos y ramas; tronco tortuoso; necrosis y exudaciones. Otros aspectos evaluados fueron: daños en las raíces generados por obras de infraestructura; presencia de grietas y rajaduras en el tronco; inclinación del tronco por la cercanía de las edificaciones o vías peatonales y vehiculares.

La identificación de los principales conflictos generados por el arbolado urbano se realizó mediante la inspección visual del área objeto de estudio, donde se evaluaron aquellas situaciones que de manera directa o indirecta inciden negativamente el desarrollo habitual de la planta, o entran en conflicto con él. 
Para el cumplimiento de unos de los objetivos específicos del presente trabajo se elaboró una encuesta como técnica de adquisición de información. Mediante la misma se pudo conocer la percepción de los miembros de la comunidad acerca del arbolado urbano. Para determinar el tamaño de la muestra se aplicó la siguiente expresión matemática, propuesta por Calero (1978):

$$
n=\frac{\frac{\left(Z_{1-\alpha / 2}\right)^{2}}{d} * P-(1-P)}{1+\frac{1}{N} *\left(\frac{Z_{1-\alpha / 2}}{d}\right)^{2} * P(1-P)-\frac{1}{N}}
$$

En este sentido, se aplicó la encuesta a 70 visitantes de los parques y espacios públicos, lo cual incluye estudiantes de la Escuela Secundaria Básica Urbana Carlos Ulloa, todos comprendidos dentro del área de estudio. La población seleccionada es heterogénea en su composición, pues deben existir muchas posibilidades y alternativas para apreciar todas las respuestas posibles con respecto al tema. Teniendo en cuenta el rango de edades de los entrevistados, se establecieron cuatro intervalos para facilitar el procesamiento, a criterio de los autores: 10 - 17 años, 18 - 25 años, 26 - 35 años y 36 años o más. Los resultados se procesaron aplicando un análisis de calidad Pareto, apilado con el paquete estadístico Statical Package for Social Science (SPSS) versión 15.0.1 2006 sobre Windows. Además, se empleó el tabulador de texto Microsoft Office Excel 2007 para realizar la base de datos de las entrevistas.

\section{RESULTADOS Y DISCUSIÓN}

A partir del inventario realizado en las áreas de estudio se determinaron un total de 94 especies distribuidas en 48 familias y 86 géneros.

En la figura 1 se destaca la distribución de las especies por hábitos de crecimiento. Predominan los árboles, especialmente las especies Delonix regia (Bojer. ex Hook.) Raf. En cuanto a los árboles estipitados solo representan el $5 \%$ del total de individuos, destacándose las especies Roystonea regia (H. B. K.) O. F. Cook y Cocos nucifera L. Entre las familias más representadas se encuentran: Bignoniaceae, Malvaceae, Meliaceae, Caesalpinaceae, Annonaceae y Arecaceae.

Figura 1. Hábitos de crecimiento de las especies.

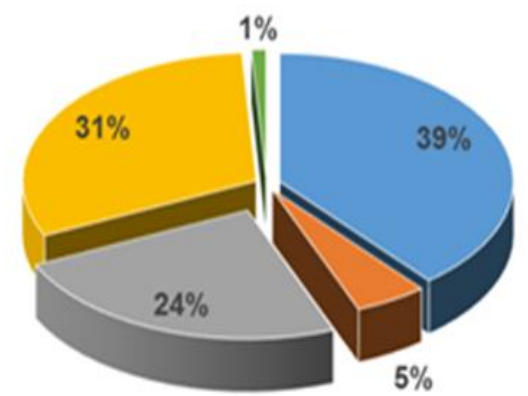

- Árbol - Árbol estipitado * Arbusto * Herbácea - Trepadora

Fuente: Elaboración propia.

Con respecto al origen geográfico (figura $2 a-2 b$ ), predominan las especies introducidas tanto para los árboles como para los arbustos. Estos resultados son similares de los obtenidos por Sosa-López et al. (2011) de un diagnóstico de la situación del arbolado urbano en la ciudad de Guisa. Del total de 
especies, el $63 \%$ para la ciudad de Guisa y el $64 \%$ para el consejo popular Hermanos Cruz, introducidas de diversos sitios del mundo. De forma general, esta problemática está presente en las diferentes ciudades del país por la fácil obtención y crecimiento de esas especies. Ello se debe a que los programas de arborización urbana que han tenido lugar en la ciudad, no han contemplado una mayor presencia de especies nativas de la región con amplias posibilidades para la ornamentación de espacios urbanos Sosa-López et al. (2011).

Figura 2a. Origen de las especies (árbol). Figura $\mathbf{2 b}$. Origen de las especies arbustivas.
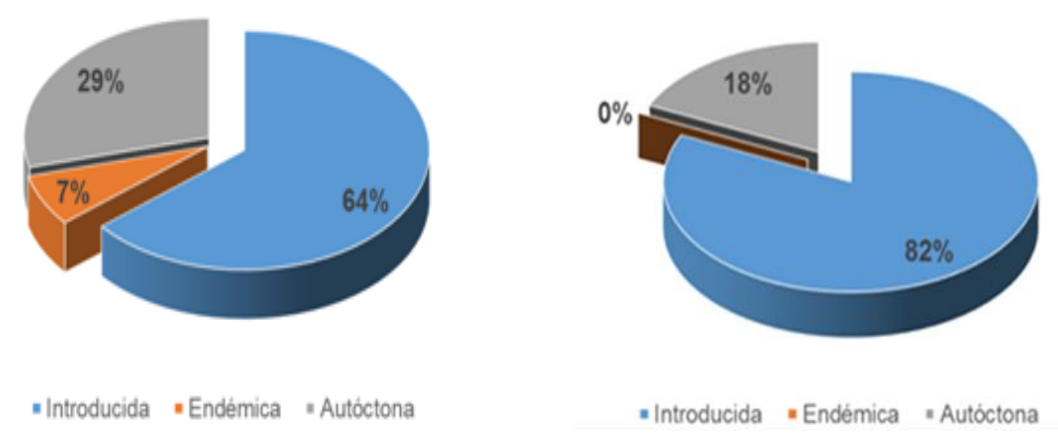

Fuente: Elaboración propia.

Con respecto al número de individuos por especies, se puede apreciar que resultan más numerosas las especies Veitchia merrillii (Becc.) H. E. Moore, Delonix regia, Eucalyptus sp. Caryota urens L., Taliparitis elatum Sw. y Pinus caribaea Morelet, de las cuales solo las dos últimas son autóctonas.

El arbolado del reparto Hermanos Cruz cuenta en su composición con $39 \%$ de árboles, $24 \%$ de arbustos, $8 \%$ de árboles estipitados, y solo un $1 \%$ de epifitas y enredaderas; esta composición no varía en función de la clasificación de las áreas verdes (figura 3).

Figura 3. Áreas verdes del reparto Hermanos Cruz.

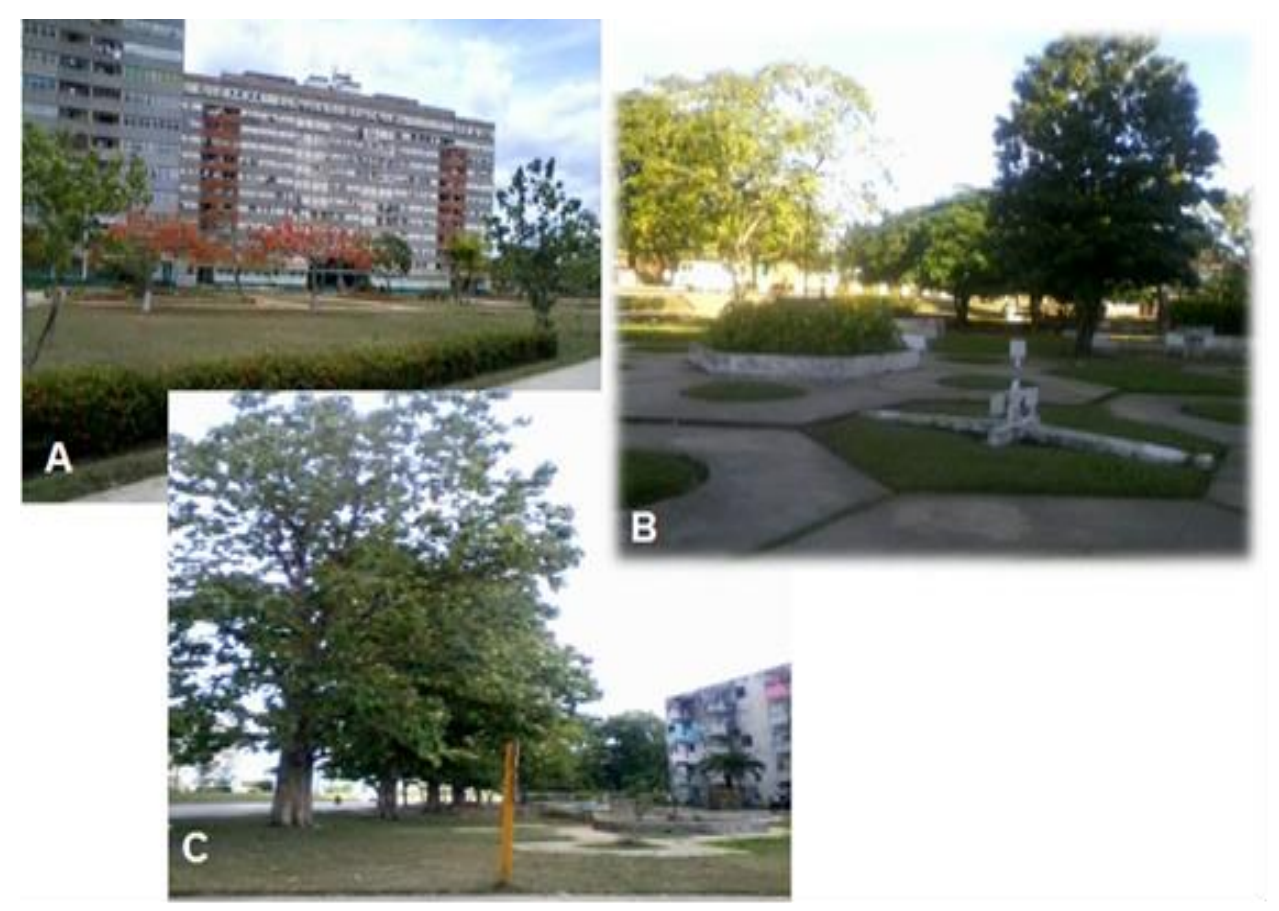

Fuente: Elaboración propia. 
En los distintos espacios verdes presentes en la zona evaluada se observó una similitud determinada por la presencia de las mismas especies entre las 11 áreas; lo cual varió solamente en el número e indica que hay un grupo de plantas que, por lo explicado anteriormente, son las que más se utilizan. Estos resultados difieren de los obtenidos por Pérez (2014) al comparar las áreas verdes de los diferentes consejos populares.

El número de especie oscila de 5 a 23 en las diferentes áreas. El de menor diversidad es el Centro comercial con cinco y el de mayor abundancia la calle Los Pinos con 23, donde se observa una gran variedad de plantas arbóreas en los jardines.

En la tabla 1 se muestra el comportamiento de las especies de mayor frecuencia y abundancia relativa, correspondientes a las áreas inventariadas; las especies que se registran con mayor frecuencia son: Delonix regia (Bojer. ex Hook.) Raf, Chrysalidocarpus lutscens W. Wendl y Veitchia merrillii (Becc.) H. E. Moore.

Tabla 1. Frecuencia relativa y la abundancia relativa de las especies más frecuentes.

\begin{tabular}{lcc}
\hline Nombre científico & Fr (\%) & Ar (\%) \\
\hline Brassaia actinophylla F.Müll & 36.36 & 2.93 \\
Cedrela odorata L. & 36.36 & 2.34 \\
Moringa oleiffera Lam. & 36.36 & 2.15 \\
Pinus caribaea Morelet & 36.36 & 5.13 \\
Psidium guajava L. & 45.45 & 2.34 \\
Taliparitis elatum Sw. & 45.45 & 5.47 \\
Chrysalidocarpus lutscens W.Wendl & 54.54 & 6.26 \\
Veitchia merrillii(Becc.) H. E. Moore & 72.73 & 19.37 \\
Delonix regia (Bojer. ex Hook.) Raf & 81.81 & 7.24 \\
\hline
\end{tabular}

Fuente: Elaboración propia.

Sobre la determinación de los árboles de riesgo, durante el recorrido por las distintas áreas de estudio, se observó que algunos exponentes presentan alteraciones en sus características morfológicas como pudrición de fustes y ramas, brotes de raíces, troncos torcidos y con cavidades. Se pudo apreciar que la cercanía de los árboles a la instalación escolar ha provocado afectaciones en su crecimiento, al no disponer estos del espacio suficiente para su desarrollo y no seleccionarse adecuadamente la especie en el momento de la plantación, lo cual puede provocar la caída de los mismos por la ocurrencia de vientos fuertes.

Se han presentado situaciones de peligro que son habituales y recurrentes en los distintos ambientes urbanos como, por ejemplo, la afectación del pavimento en parques cercanos a edificios, debido a la inadecuada selección de plantas, principalmente en las vías de circulación.

En dichas áreas, tanto viales y peatonales, se pudo apreciar la interferencia de las ramas de los árboles con el tendido eléctrico, lo cual puede ocasionar accidentes en caso de vientos fuertes o huracanes. Esta problemática se debe a que el personal especializado para la poda y desmoches "autorizados» de las entidades de servicios públicos, no se ha interesado por darle una solución a ello y, en ocasiones, no se realizan esa actividad con la calidad técnica requerida. 


\section{Resultados de las encuestas}

Del total de los encuestados, 43 corresponden al sexo masculino, en tanto que al femenino 27 . Otra variable estudiada fue la ocupación con 22 estudiantes, 17 trabajadores (15 de ellos con nivel universitario), nueve técnicos, cuatro cuentapropistas, dos jubilados y una ama de casa. El comportamiento fue el siguiente:

- Pregunta 1. ¿Considera necesaria la presencia de los árboles en las ciudades? ¿Por qué?

Con la interrogante se evalúa el nivel de conocimientos de la población al clasificarse sus argumentos en respuesta avanzada, intermedia o negativa (figura 4). A pesar de que la mayoría de los estudiantes dieron respuestas intermedias y la minoría respuestas avanzadas, en su conjunto han demostrado estar orientados con respecto a la función y los beneficios del arbolado; y consideran necesaria la existencia de especies arbóreas en su entorno citadino. Ello se debe no solo a que las asignaturas impartidas están relacionadas con estos temas, a lo que sino también a las charlas educativas y debates sobre el medio ambiente.

Para el caso de los universitarios hubo coincidencia en las respuestas de ambas clasificaciones, apreciándose que conocen del tema y lo consideran importante. Los técnicos, trabajadores, cuentapropistas, jubilados y amas de casa, en su totalidad, ofrecieron respuestas intermedias, en correspondencia con la información que le proporcionan los medios de difusión masiva, y expresaron que los árboles son «los pulmones de la ciudad».

Figura 4. Resultados de la pregunta 1 en función de las ocupaciones.

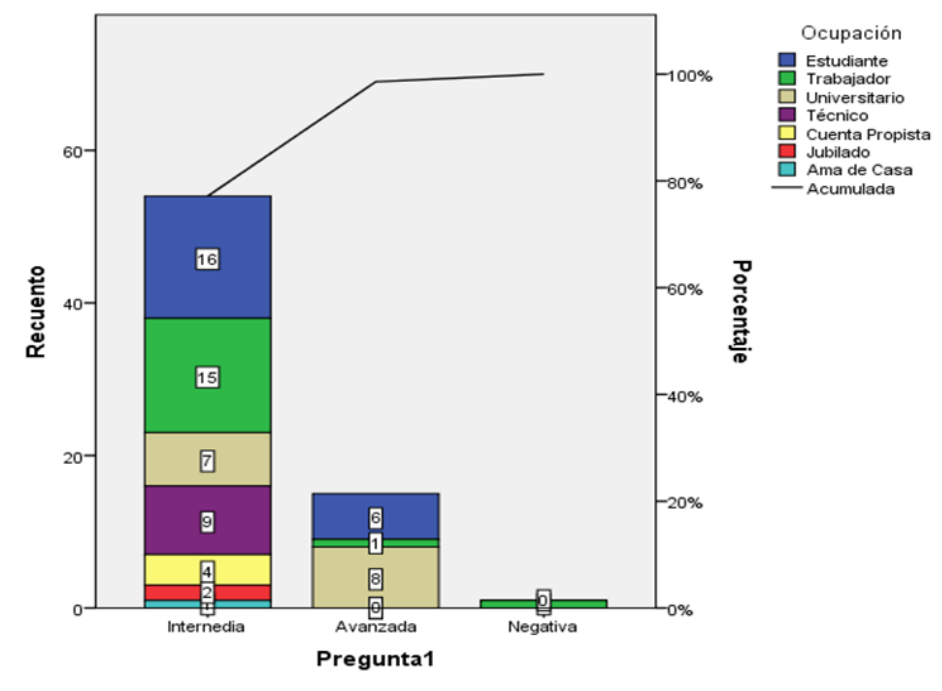

Fuente: Elaboración propia.

\section{- Pregunta 2. ¿Cómo considera el número de árboles en su comunidad?}

Con respecto a la edad el $20 \%$ consideran abundante el número de especies, en tanto que el $50 \%$ y el $30 \%$ la clasifican como regular y escasa respectivamente en su entorno citadino. Esto demuestra que no existe una adecuada cantidad de árboles en los espacios verdes, problemática que pudiera ser ocasionada por diversas razones, tales como la falta de coordinación y adecuada jerarquización de las tareas y responsabilidades entre los encargados de los verdes urbanos, o la inadecuada gestión y escasez de especies en los viveros. Esos resultados difieren de los reportados por Perdomo (2015) en un estudio realizado en San Nicolás, municipio de la provincia Mayabeque, el cual expresa que los 
jóvenes no perciben la necesidad de los árboles para la salud de los pobladores y el embellecimiento de la localidad.

- Pregunta 3. ¿Dónde le gustaría que hubiera presencia de arbolado?

Los encuestados podían optar por más de una respuesta. Los hombres han preferido la presencia de arbolado principalmente en los parques, jardines y calles, mientras que las mujeres optan por la existencia de árboles en los parques y jardines solamente.

- Pregunta 4. ¿Qué especies de árboles le gustaría encontrar en su entorno?

Un número predominante de encuestados prefieren árboles frondosos que proyectan buena sombra. Las especies más repetidas son: Taliparitis elatum, Pinus caribaea, Cedrela odorata L., Terminalia catappa L., Swtienia macrophylla King., Mangifera indica L., Delonix regia y Tabebuia angustata L. Un total de 23 encuestados no respondieron por desconocimiento de las especies.

- Pregunta 5. ¿Qué utilidad le atribuye al arbolado urbano?

En esta interrogante se abrevaron varios puntos de vistas, pero en su generalidad la mayoría de los encuestados coinciden en que el arbolado es una parte del paisaje que hace más atractiva a la ciudad, a la vez que consideran que es una parte esencial de la infraestructura de la misma (figura 5).

Figura 5. Percepción de los encuestados sobre las características del arbolado urbano.

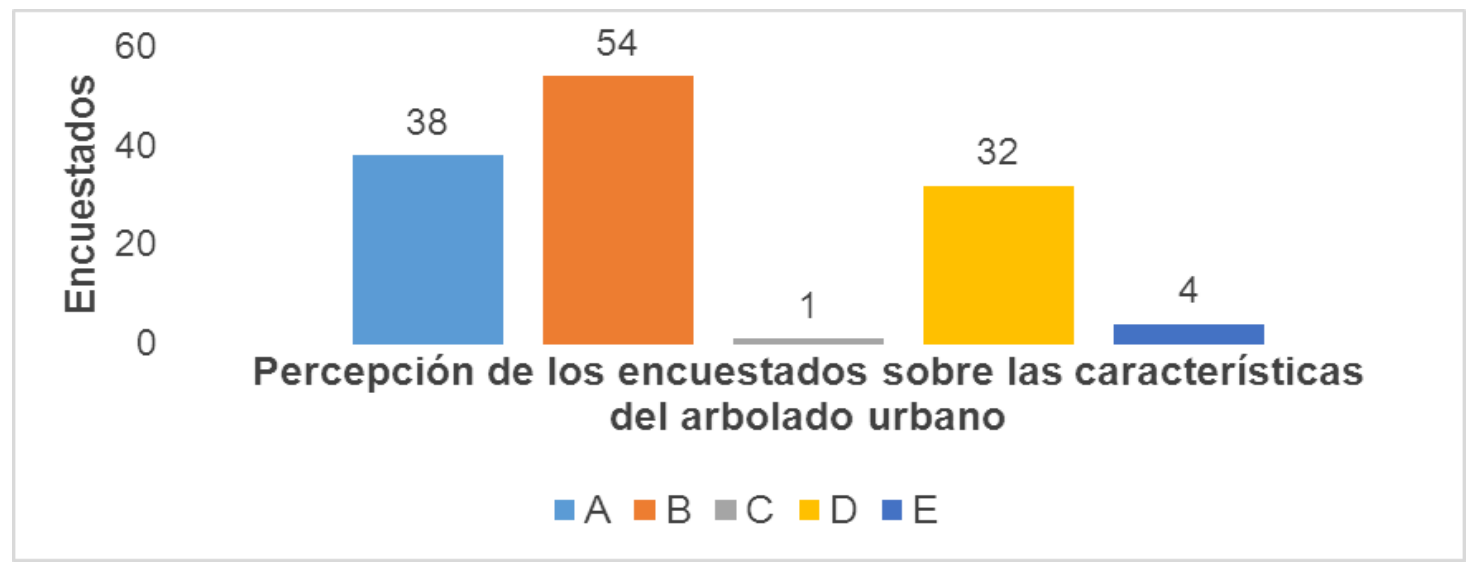

Nota: A. Es una parte esencial de la infraestructura de la ciudad, B. Una parte del paisaje que hace a la ciudad más atractiva. C. Algo innecesario que solo estorba. D. Algo útil si se le da el cuidado necesario. E. No sabe. Fuente: Elaboración propia.

\section{CONCLUSIONES}

En las áreas verdes del reparto Hermanos Cruz predominan las especies exóticas en los diferentes estratos y se hace necesario incrementar la presencia de especies autóctonas.

La no adecuada selección de las especies lleva a la presencia de árboles de riesgo, por lo que se requiere de la aplicación de podas o talas, según el nivel de afectación.

Los encuestados consideran necesaria la presencia de árboles en las ciudades y demuestran conocimientos acerca del arbolado en su entorno citadino, en cuanto a sus funciones y beneficios. 


\section{REFERENCIAS BIBLIOGRÁFICAS}

Acevedo, P. y Strong, M.T. (2012). Catalogue of Seed Plants of the West Indies. Smithsonian contributions to botany, (98). Smithsonian Institution Scholarly Press https://doi.org/10.5479/si.0081024X.98.1

Álvarez de Zayas, A. (2008). Plantas ornamentales en Cuba: usos, diversidad y amenazas. Revista del Jardín Botánico Nacional, (29), 83-100. https://www.jstor.org/stable/42597271?seq=1

Bisse, J. (1988). Árboles de Cuba. Editorial Científico -Técnica.

Chang, J.A. (14 al 17 de abril 2015). Diagnóstico espacial de la cobertura de copas del arbolado público en la ciudad de Palma Soriano [Ponencia]. Convención Internacional Agroforestal 2015. La Habana, Cuba.

Calero, A. (1978). Técnica de muestreo. Editorial Pueblo y Educación.

Castillo, L. y Ferro, S.A. (2015). La problemática del diseño con árboles en vías urbanas: "verde con pespuntes negros". Arquitectura y Urbanismo, 36(1), 5-24. https://n9.cl/tn41

Iguiñiz, G. (2007). Apuntes de Gestión de la estructura del arbolado urbano [Archivo PDF]. https://www.jardin-natural.com/wp-content/uploads/2017/12/gestdelaestructura2.pdf

Ministerio de Ciencia, Tecnología y Medio Ambiente (1978). Áreas verdes (NC 53-05:78). La Habana, Cuba.

Ministerio de Ciencia, Tecnología y Medio Ambiente (1987). Áreas verdes urbanas. Términos y definiciones (NC 93-06-101:87). La Habana, Cuba.

Pérez, M. (2014). Evaluación de las condiciones ambientales de los espacios públicos de Pinar del Río [Tesis de maestría no publicada]. Universidad de Pinar del Río.

Perdomo, D. (2015). El arbolado urbano en San Nicolás: Una visión desde la gestión del conocimiento. [Ponencia]. Convención Internacional Agroforestal 2015. La Habana, Cuba

Sablón, A.M. (1987). Dendrología. Editorial Científico Técnica.

Sosa-López, A., Molina- Pelegrín, Y., Puig-Pérez, A. y Riquenes-Valdés, A. (2011). Diagnóstico de la situación del arbolado urbano en la ciudad de Guisa. Revista Forestal Baracoa, 30(1), 73-78. 